\title{
MECHANICAL PROPERTIES AND SOUND VELOCITY OF GOLD COPPER (AuCu) II SUPERLATTICE: 3D MOLECULAR DYNAMIC (MD) SIMULATION
}

\author{
Mohammed M. Aish \\ Physics Department Menoufia University, Gamal Abd El-Nasir, Menoufia, Egypt \\ e-mail: mohamedeash2@yahoo.com
}

\begin{abstract}
Young's modulus, yield stress and Poisson's ratio are studied for different size and temperature. The temperature dependence of simulated Young's modulus is quite similar to experimental results. Transverse sound velocity is estimated from the simulated elastic constants at each temperature. The dislocation speed reaches up to $75 \%$ of the transverse sound velocity. The dislocation speed decreases with increasing temperature linearly. The temperature dependence of macroscopic deformation behavior and the possibility of the existence of supersonic dislocations are discussed. The transverse sound velocity and rigidity $G$ is calculated from Young's modulus, Poisson's ratio and density $\rho$ which changes with temperature.
\end{abstract}

Keywords: sound velocity, rigidity, Young's modulus, Poisson's ratio, density

\section{Introduction}

$\mathrm{AuCu}$ II is a one-dimensional long-period ordered structure based on $\mathrm{AuCu}$ I structure, which has anti-phases with shifts of $0,1 / 2,1 / 2$, and its unit cell corresponds to 10 unit cells of $\mathrm{AuCu}$ I. $\mathrm{Au}-\mathrm{Cu}$ alloys exhibit excellent mechanical strength, chemical stability, and process ability. Thus, they are employed as dental materials, jewelry and industrial materials. At high temperatures, these types of alloys exist in the form of solid solutions over the entire range of compositions. It is well known, however, that they form ordered alloys below a certain transition temperature which depends on the alloy composition. The order-disorder transition of a stoichiometric $\mathrm{AuCu}$ alloy (hereafter referred to as $\mathrm{AuCu}$ alloy) has been extensively studied. The ordered phase is stable at low temperatures and has a face-centered tetragonal structure (hereafter referred to as $\mathrm{AuCu} \mathrm{I).}$

Heat treatment can be used to harden gold-copper alloys by a slightly different process. Because the gold-copper system forms a complete solid solution at all compositions, the atoms can be interchanged on the lattice sites. However, as the atoms are cooled, the copper and gold atoms tend to separate on alternating planes of the lattice in an ordered arrangement. This ordering makes dislocation motion more difficult, raising the yield stress. The Zener relaxation in yellow gold alloys (of sufficiently high copper content) exhibits the same characteristics. These materials harden because they form an ordered phase of tetragonal symmetry like AuCu.

Molecular dynamics simulations have been applied successfully in many fields including chemical physics, materials science and in the modeling process of biomolecules. Comprehensive reviews of the subject can be found in literature (Haile, 2001; Leach, 2001; Schlick, 2002; Griebel et al., 2007). The mechanical properties of Ni nanowires are highly dependent on size, shape as well as atomic vacancies in the nanowire were shown in (Aish, 2019). Structural transformation and mechanical properties of Ti nanowire were studied in (Aish and Starostenkov, 2016). Characterization of strain-induced structural transformations in CdSe nanowires using molecular dynamics simulation was given in (Aish et al., 2015). Mechanical properties of nanowires using nano indentation were studied by Antohe et al. (2001). 
The yield strength decreases with increasing temperature. Low yield strength at high temperature is because of the fact that thermal fluctuations which overcome the energy barrier for dislocation nucleation. Detailed MD results concerning the temperature dependence of the core structure have been obtained.

MD simulation has been used to investigate the effect of temperature and cell size of $\mathrm{AuCu}$ II superlatticeon explaining the nature of deformation and fracture. The engineering stress and strain dependent on temperature and size of the simulated cell obtained by the MD of tensile specimens. The nature of deformation, Young modulus, yield stress, yield strain and Poisson's ratio were studied. MD simulation of deformation under a uniaxial tensile strain condition for $\mathrm{AuCu}$ II alloys was performed in order to clarify the temperature and size dependence. Simulations are performed for the temperature range from $100 \mathrm{~K}$ to $500 \mathrm{~K}$ using Cleri-Rosata potentials. Structural changes taking place in nanowires of $\mathrm{AuCu}$ II alloys during mono-axial tension investigated in the course of high-rate, tensile uniaxial loading along $\langle 001\rangle$ and $\langle 100\rangle$ at different temperatures and cell size were studied. The presence of periodic thermal planar defects in the long-period nanostructure (combined thermal anti-phase boundaries) significantly affects the onset of plastic deformation.

The object of investigation are nanowires of $\mathrm{AuCu}$ II superlattice alloy. We study the effect of temperature and size of the simulated cell on mechanical properties of simulated nanowires.

\section{The MD method}

MD simulations were used to simulate movement of each atom of interacting atoms based on Newtonian mechanics. Given instantaneous position and velocity vectors of the atoms, dynamical history of the composition was generated by numerically integrating equations of motion. The force and energy of the system were calculated, atoms in the composition were given in the Cartesian coordinate components for different condition of thermodynamics and kinetics. The computer simulation process allowed for the equilibrium system until minimal interatomic energy and force through development of suitable interatomic potentials.

When modeling deformation in nanowires studied, there was a periodic repetitive progressive shift of atoms which make up absolutely rigid grips along the axis of stretching nanowires in opposite directions from each other. The structure of absolutely rigid grips remained unchanged throughout the experiment. Non-deformable absolutely rigid grips were shifted by $0.001 \mathrm{~nm}$ every $0.1 \mathrm{ps}$. The total speed of the atoms was $20 \mathrm{~m} / \mathrm{s}$ and corresponded to a strain rate of $2.29 \cdot 10^{9}$ to $3.39 \cdot 10^{9} \mathrm{~s}^{-1}$ depending on length of the investigated nanowire. This order of velocities is found in works on modeling tensile/compression strains. At the beginning of a computer experiment, the temperature was set through the velocities of atoms, the modules of which were calculated by the formula

$$
\left|v_{i}\right|=\sqrt{\frac{2 k_{b} T_{0}}{m_{i}}}
$$

where $k_{b}$ is the Boltzmann constant, $T_{0}$ - set temperature, $m_{i}$ - atomic mass of atom $i$.

During deformation of the nanowire, thermostatting was performed with the reaction time of the thermostat $t_{r}=0.1 \mathrm{ps}$ according to the Berendsen algorithm. The structural rearrangement of atoms inside the calculated block of the crystal was realized by the molecular dynamics method by solving a system of ordinary differential equations of Newton's motion that describes the motion of particles.

Molecular dynamics simulation requires a definition of a potential function or a description of terms by which the particles in the simulation will interact. In chemistry and biology this is usually referred to as a force field, and in material physics as an interatomic potential. Potentials 
may be defined at many levels of physical accuracy; those most commonly used in chemistry are based on molecular mechanics and embody classical mechanics treatment of particle-particle interactions that can reproduce structural and conformational changes but usually cannot reproduce chemical reactions.

$\mathrm{Ab}$ initio, quantum mechanical and chemical methods may be used to calculate potential energy of a system on the fly, as needed for conformations in a trajectory. This calculation is usually made in the close neighborhood of the reaction coordinate. Although various approximations may be used, these are based on theoretical considerations, not on empirical fitting. $\mathrm{Ab}$ initio calculations produce a vast amount of information that is not available from empirical methods, such as density of electronic states or other electronic properties. A significant advantage of using ab initio methods is the ability to study reactions that involve breaking or formation of covalent bonds, which correspond to multiple electronic states.

To make the nanowires in the state with the lowest energy we applied the dynamic relaxation method of molecular dynamics. The interaction of atoms in the alloy was set by many-tight-binding potential Clery-Rosato (Cleri and Rosato, 1993)

$$
E=\sum_{i}\left(-\sqrt{\sum_{i \neg j} \xi_{\alpha \beta}^{2} \exp \left(-2 q_{\alpha \beta} \frac{r_{i j}}{r_{0}^{\alpha \beta}-1}\right)}+\sum_{i \neq j} A_{\alpha \beta} \exp \left(-p_{\alpha \beta} \frac{r_{i j}}{r_{0}^{\alpha \beta}-1}\right)\right)
$$

where $\xi, A$ are energy parameters corresponding to the properties (depth) of the potential well, $q, p$ - parameter determining stiffness of interatomic bonds, $r_{0}$ - average equilibrium distance which takes into account the interaction between the atoms. Dynamic relaxation was carried out at an initial temperature of $0 \mathrm{~K}$ for a $10 \mathrm{ps}$. The nanowires can be heated to temperatures of $100 \mathrm{~K}$ (but not more than $120 \mathrm{~K}$ ), due to release of energy. The strain was set along each of the axes alternately, i.e. it is uniaxial. The interval defining deformation ranged within $-12 \%$ to $12 \%$ depending on the considered axis. The calculation unit was kept at a predetermined deformation temperature of $0.6 \mathrm{Tm}$ for $0.05 \mathrm{~ns}$, then cooled every $0.001 \mathrm{ps}$.

This work was performed using our new MD program "Structural Transformation of Metallic FCC, BCC and HCP Nanowires and Their Alloys Using Cleri-Rosato potential" (Aish, 2019). The interatomic interactions were calculated using the tight-binding potentials (Cleri and Rosato, 1993). We used a simpler, more efficient computer program for simulating a system involving several thousands of atoms in the time interval up to several nanoseconds.

Since the nature of deformation, Young's modulus, yield stress, yield strain and Poisson's ratio of $\mathrm{AuCu}$ II superlattice nanowires are of main interest in this work (Tables 1 and 2), it seems to be reasonable to adopt small $L_{x}, L_{y}$ and $L_{z}$ in simulations. To save the computing time, the dimensions of the MD models used in the following simulations are set to be $L_{x}=L_{y}=L_{z}$, and $L_{z}$ changes from 5 to 30 atomic plane. The nature of deformation, Young's modulus, yield stress, yield strain and Poisson's ratio of AuCu II superlattice were studied.

Table 1. Typical molecular dynamic simulation results of $\mathrm{AuCu}$ II superlattice for different cell size at room temperature, Young's modulus $E[\mathrm{GPa}]$, tensile strength, tensile strain, Poisson's ratio and rigidity $G$

\begin{tabular}{|c|c|c|c|c|c|}
\hline $\begin{array}{c}L_{z} \\
{[\mathrm{~nm}]}\end{array}$ & $\begin{array}{c}E \\
{[\mathrm{GPa}]}\end{array}$ & $\begin{array}{c}\text { Yield stress } \\
{[\mathrm{GPa}]}\end{array}$ & $\begin{array}{c}\text { Yield strain } \\
{[\%]}\end{array}$ & $\begin{array}{c}\text { Poisson's ratio } \\
{[\%]}\end{array}$ & $\begin{array}{c}G \\
{[\mathrm{GPa}]}\end{array}$ \\
\hline \hline 1.8 & 81.2 & 11.91 & 0.143 & 0.11 & 45.617 \\
\hline 3.6 & 86.1 & 11.8 & 0.131 & 0.178 & 52.372 \\
\hline 5.4 & 94 & 12.35 & 0.1305 & 0.14 & 54.651 \\
\hline 7.2 & 93 & 10.73 & 0.116 & 0.102 & 51.781 \\
\hline 9.4 & 93.1 & 10.35 & 0.109 & 0.102 & 51.837 \\
\hline
\end{tabular}


Table 2. Typical molecular dynamic simulation results of $\mathrm{AuCu}$ II superlattice for two cell size (10 and 30 atomic plane with cubic form) at different temperature, Young's modulus E [GPa], tensile strength, tensile strain and Poisson's ratio

\begin{tabular}{|c|c|c|c|c|c|c|c|c|}
\hline \multirow{2}{*}{$T[\mathrm{~K}]$} & \multicolumn{2}{|c|}{$E[\mathrm{GPa}]$} & \multicolumn{2}{|c|}{ Yield stress [GPa] } & \multicolumn{2}{c|}{ Yield strain [\%] } & \multicolumn{2}{c|}{ Poisson's ratio [\%] } \\
\cline { 2 - 9 } & 30 & 10 & 30 & 10 & 30 & 10 & 30 & 10 \\
\hline \hline 100 & 99 & 97 & 12.7 & 15.1 & 0.153 & 0.123 & 0.162 & 0.111 \\
\hline 200 & 96.1 & 78 & 12.6 & 11.7 & 0.1405 & 0.1312 & 0.20 & 0.125 \\
\hline 300 & 94 & 82 & 12.35 & 11.95 & 0.14305 & 0.131 & 0.11 & 0.14 \\
\hline 400 & 89 & 65 & 11.73 & 10.7 & 0.128 & 0.133 & 0.118 & 0.14 \\
\hline 500 & 83.1 & 74 & 10.85 & 11.5 & 0.119 & 0.13 & 0.072 & 0.1398 \\
\hline
\end{tabular}

\section{The nature of mechanical properties}

\subsection{The nature of mechanical properties at room temperature}

In this part, $\mathrm{MD}$ simulations are performed for the $\mathrm{AuCu}$ II superlattice alloy nanowire subject to uniaxial tensile strain loading. Deformations of the nanowire are in a fast stage of the atomic damage process. The mechanical properties at different volumes of simulated lattice with MD simulations, the uniaxial tension of $\mathrm{AuCu}$ II superlattice nanowires are studied for different nanowire volumes at $300 \mathrm{~K}$.

Figure 1 shows the change of Young's modulus with the cell size. The trend of size dependence of Young's modulus of the simulated results is quite similar to other findings (Aish, 2019).

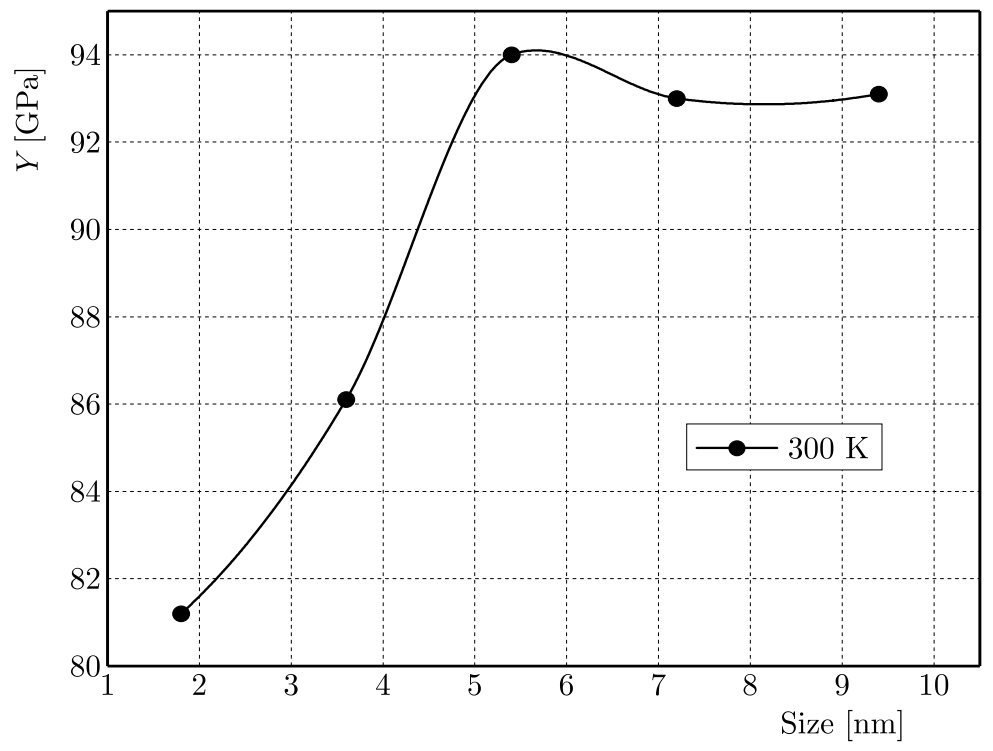

Fig. 1. Relation between Young's modulus with the cell size at $300 \mathrm{~K}$

In Fig. 2, the change of yield stress at different size cell is shown. The trend of size dependence of yield stress of the simulated results is divided into three regions; in the first region from $1 \mathrm{~nm}$ to $3.6 \mathrm{~nm}$ the yielding stress seems to be constant. In the second region from $3.6 \mathrm{~nm}$ to $5.5 \mathrm{~nm}$, the yielding stress increases. Finally, in the third region from 5.5 up to $10 \mathrm{~nm}$, the yielding stress decreases with increasing size of the cell (Aish, 2019).

Figure 3 shows the change of yield strain with the cell size. The trend of size dependence of yield strain of the simulated results is shown; the yield strain drastically decreases with the increasing cell size. 


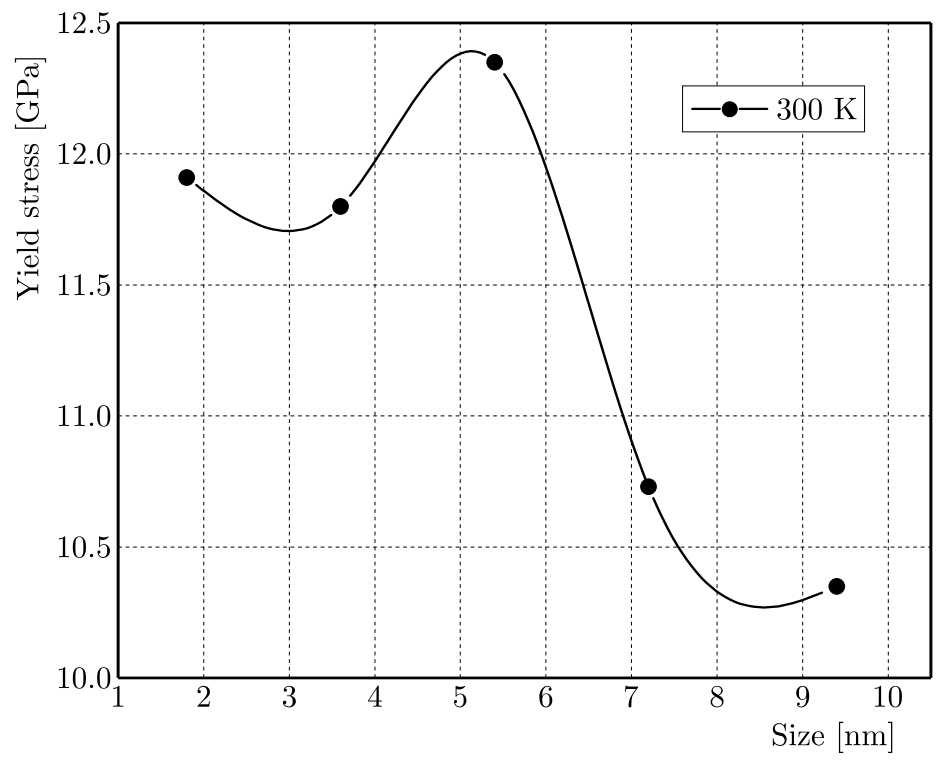

Fig. 2. Relation between the yield stress and the cell size at $300 \mathrm{~K}$

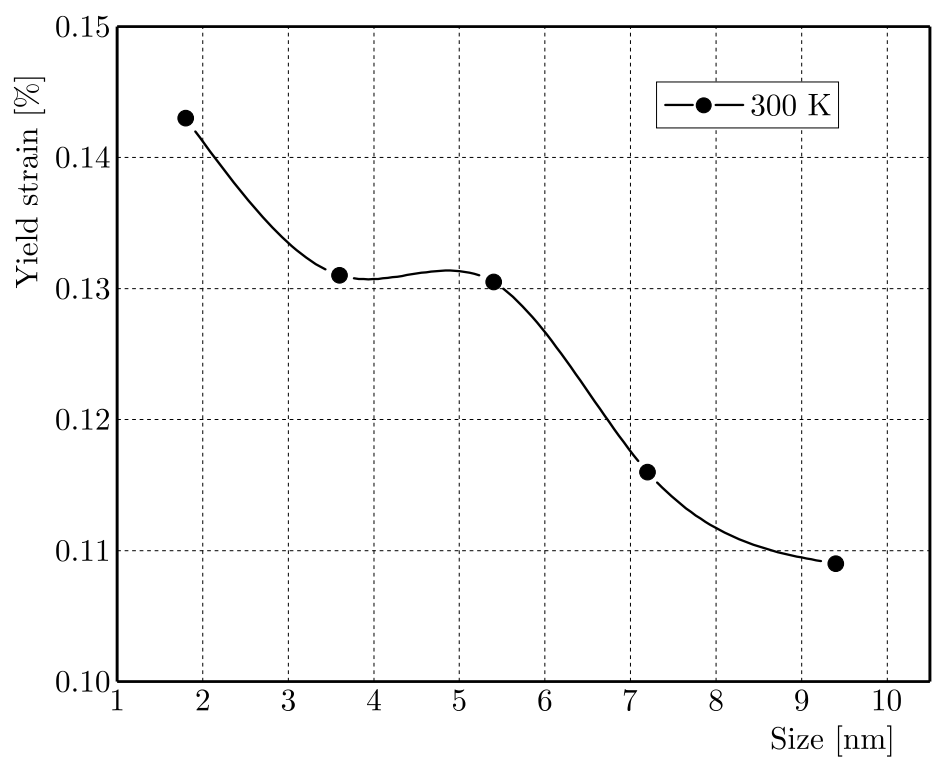

Fig. 3. Relation between yielding stress with size cell at $300 \mathrm{~K}$

In Fig. 4, the change of Poisson's ratio with different size cell is shown. The trend of size dependence of Poisson's ratio of the simulated results is divided into two regions. In the first region from $1 \mathrm{~nm}$ to $3.5 \mathrm{~nm}$, Poisson's ratio increases with increasing cell size. In the second region from 3.6 up to $10 \mathrm{~nm}$, Poisson's ratio decreases with increasing size of the cell.

\subsection{The nature of mechanical properties for the cubic form at different temperatures}

The mechanical properties by MD simulations under uniaxial tension of $\mathrm{AuCu}$ II superlattice nanowires were studied at various temperatures from $0 \dot{\mathrm{K}}$ to $500 \mathrm{~K}$ for two different sizes $(10 \times 10 \times 10$ and $30 \times 30 \times 30$ atomic plane $)$.

The results demonstrated that Young's modulus, tensile strength, tensile strain, Poisson's ratio depended on both temperature and size of the simulated cell.

Figure 5 shows the change of Young's modulus with temperature for two cell sizes. The trend of temperature dependence of Young's modulus is quite similar for different cell sizes. It decreases 


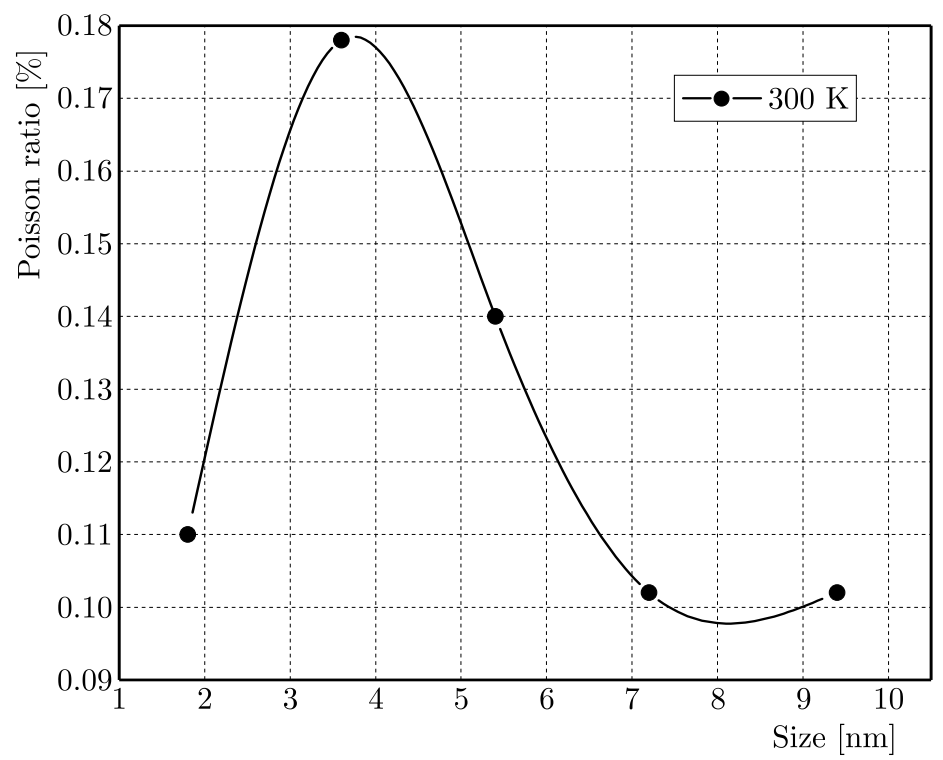

Fig. 4. Relation between Poisson's ratios with the cell size at $300 \mathrm{~K}$

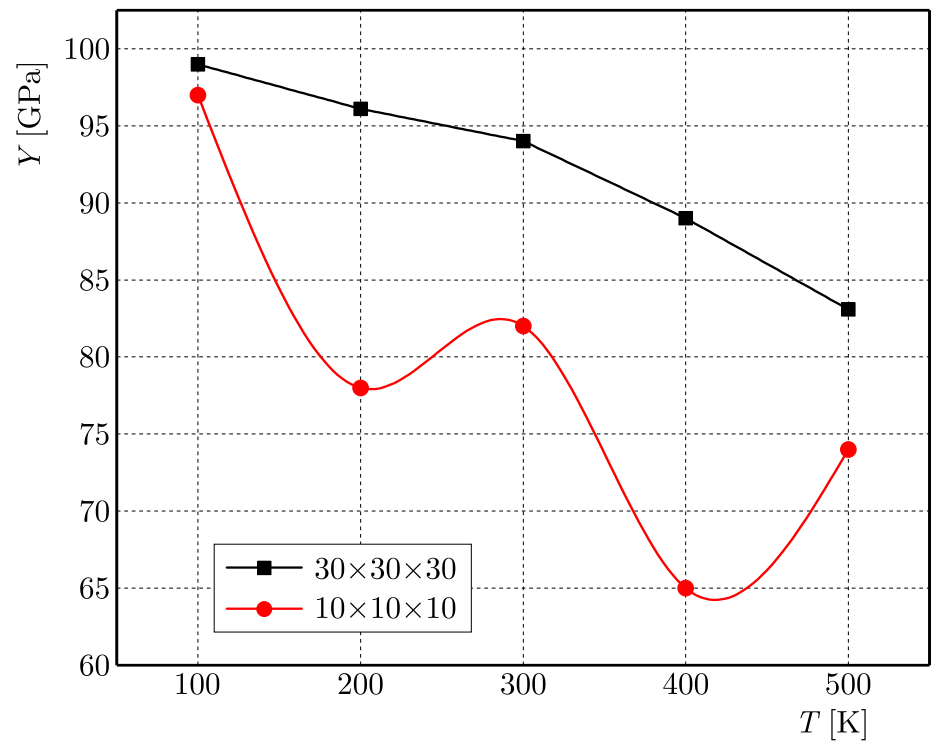

Fig. 5. Relation between Young's modulus with temperature for two different cell sizes

with increasing temperature in the numerical experiment. In Fig. 5, the change of Young's modulus for different temperatures is shown. As can be seen from the figure, simulated Young's modulus decreases with increasing temperature. Bigger Young's modulus at low temperature indicates that the nanowire maintains its elastic deformation for a long time. Whereas, smaller Young's modulus at high temperature illustrates that the plastic deformation begins quickly.

In Fig. 6, the change of yield stress at different temperatures is shown. As can be seen from the figure, the simulated stress decreases with increasing temperature. The trend of temperature dependence of the yield stress is quite similar to experimental results for $\mathrm{AuCu}$ in the temperature range from $100 \mathrm{~K}$ to $500 \mathrm{~K}$. Analysis of the results shows that the value of yield strength for $30 \times 30 \times 30$ nanowires are 1.02 at $100 \mathrm{~K}$ and 1.36 at $400 \mathrm{~K}$ times the corresponding values for $10 \times 10 \times 10$ nanowires, which is consistent with the ratio of bulk moduli of elasticity for these materials. The values of yield strength coincide with the data obtained in the framework of other models. With an increase in the temperature, a decrease in the value of yield strength 
of nanowires is observed. Figure 6 shows a decrease in strength with increasing temperature at the strain rate of $2 \cdot 10^{8} \mathrm{~s}^{-1}$ for all temperatures.

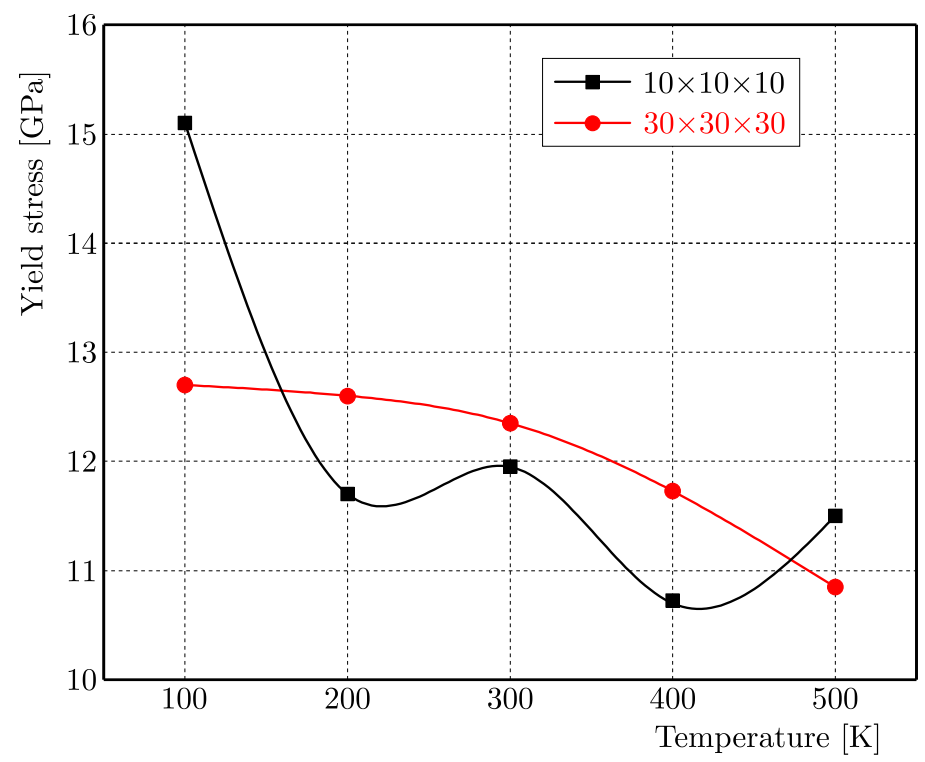

Fig. 6. Relation between yield stress and temperature for different sizes

Figure 7 shows the change of yield strain with temperature. The trend of temperature dependence of yield strain is shown; the yield strain drastically changes at different temperatures and cell sizes. The first yield strain $\varepsilon$ decreases when temperature increases. The bigger strain at low temperature indicates that the nanowire maintains its elastic deformation for a long time. Whereas, smaller strain at high temperature illustrates that the plastic deformation begins quickly.

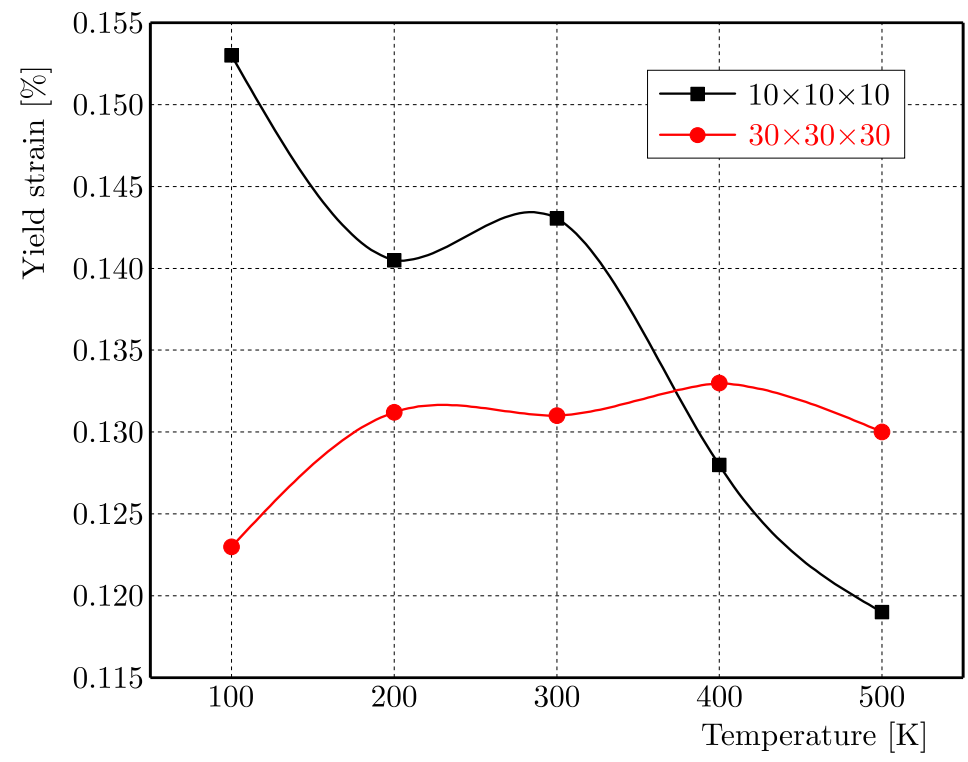

Fig. 7. Relation between yield strain and temperature experiment for different sizes

In Fig. 8, the change of Poisson's ratio at different temperatures is shown for two cell sizes. We see that as temperature and cell size increase, Poisson's ratio decreases. The reason behind the decrease in Poisson's ratio with an increase in temperature might be due to the dependence of dislocation motion on temperature. During deformation of the nanowire, the dynamic hardening 
of the material as a result of nucleation of dislocations and recovery due to climb of dislocations might be occurring simultaneously.

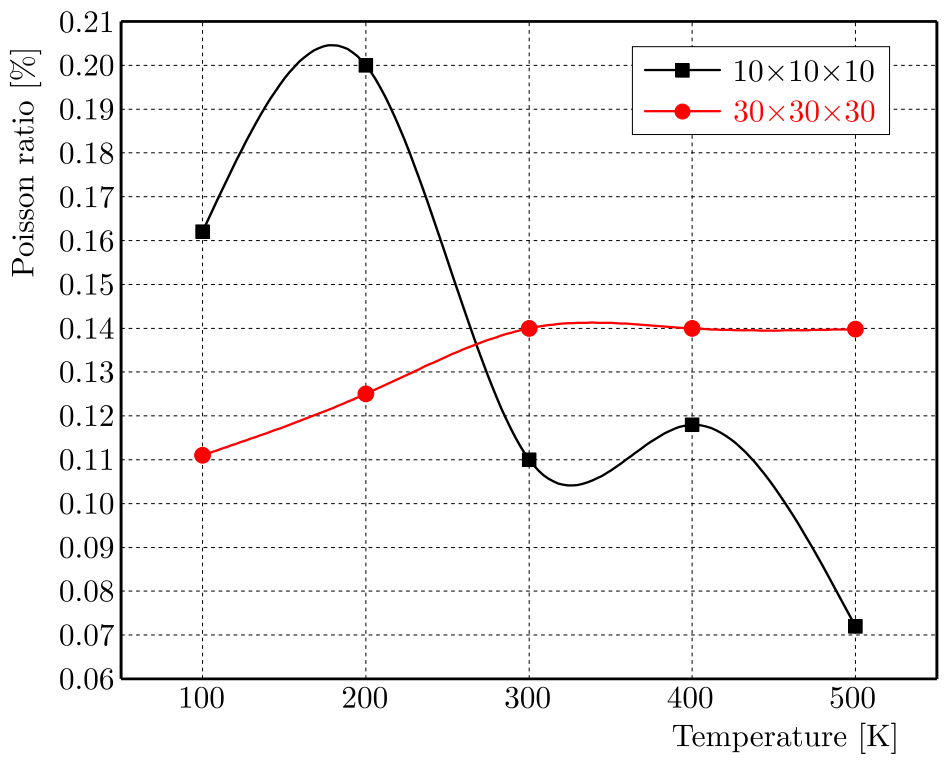

Fig. 8. Relation between Poisson's ratio with temperature for different sizes

\subsection{Calculation of sound velocity from the simulated elastic constant}

The transverse sound velocity $v_{s}^{\mathrm{T}}$ is estimated from the rigidity $G$ and density $\rho$ by, $v_{s}^{\mathrm{T}}=\sqrt{G / \rho}$. The rigidity is calculated from Young's modulus and Poisson's ratio $\nu$ by $G=E / 2(1-\nu)$. The velocity of sound in anisotropic materials is directionally dependent. Three wave velocities may exist in any given direction: two transverse waves (primary oscillation perpendicular to the wave propagation direction) and one longitudinal wave (polarization in the same direction as the propagation). You can select the type of wave (transverse or longitudinal) and whether to view the velocity surface or the inverse velocity (slowness). The contribution can either be from elastic effects alone or from elastic and piezoelectric effects. You can choose several different materials. The velocity $v$ of sound in materials depends on the elastic constants $c$ and density $\rho$. Velocity calculations can often be very simple, but because of anisotropy of many materials the elastic constant $c$ is actually a $6 \times 6$ matrix of values. Determination of the velocity of the wave requires the Christoffel equation. This description also considers the contribution of the piezoelectric coupling.

\section{Conclusion}

Metallic $\mathrm{AuCu}$ II superlattice nanowires show great potential for applications in miniaturization of electronic devices due to extraordinary mechanical strength and electrical properties. Experimental investigations of these properties are difficult because of their size and complications in performing experiments at such length scales. Computational techniques based on classical molecular dynamics simulations (using 3DMD program) provide an effective mean to understand the mechanical deformation behavior of such nanowires with considerable accuracy and predictability. In the present investigation, we have discussed the sound velocity in $\mathrm{AuCu}$ II superlattice nanowires at various temperatures and sizes due to tensile loading using classical molecular dynamics simulations (3DMD program). The effect of size and temperature on the yield strength, Young's modulus and Poisson's ratio of the nanowire has been studied in detail. 


\section{References}

1. Aish M.M., 2019, The structural transformation and mechanical strength of Ni, Ti nanowires and Nitinol alloys at various vacancy rates: molecular dynamic study using Cleri-Rosato potential, Materials Physics and Mechanics, 42, 211-223, DOI: 10.18720/MPM.4222019_8

2. Aish M.M., Shatnawi M.T.M., Starostenkov M.D., 2015, Characterization of strain-induced structural transformations in CdSe nanowires using molecular dynamics simulation, Materials Physics and Mechanics, 24, 4, 403-409

3. Aish M.M., Starostenkov M.D., 2016, Features of structural transformations of HCP metallic Ti nanowires using Cleri-Rosato potential at low temperature, Letters on Materials, 6, 4, 317-321, DOI: $10.22226 / 2410-3535-2016-4-317-321$

4. Antohe S., Ion L., Ruxandra V., 2001, Electrical properties of electron irradiated thin polycrystalline CdSe layers, Journal of Applied Physics, 90, 5928, DOI: 10.1063/1.1416141

5. Cleri F., Rosato V., 1993, Tight-binding potentials for transition metals and alloys, Physical Review $B, 48,22$

6. Griebel M., Knapek S., Zumbusch G., 2007, Numerical Simulation in Molecular Dynamics, Berlin, Heidelberg: Springer, ISBN 978-3-540-68094-9

7. Haile J.M., 2001, Molecular Dynamics Simulation: Elementary Methods, Wiley, ISBN 0-47118439-X

8. LeACH A., 2001, Molecular Modelling: Principles and Applications, 2nd edit., Prentice Hall, ISBN 978-0-582-38210-7

9. Schlick T., 2002, Molecular Modeling and Simulation, Springer, ISBN 0-387-95404-X

Manuscript received October 24, 2019; accepted for print February 21, 2020 